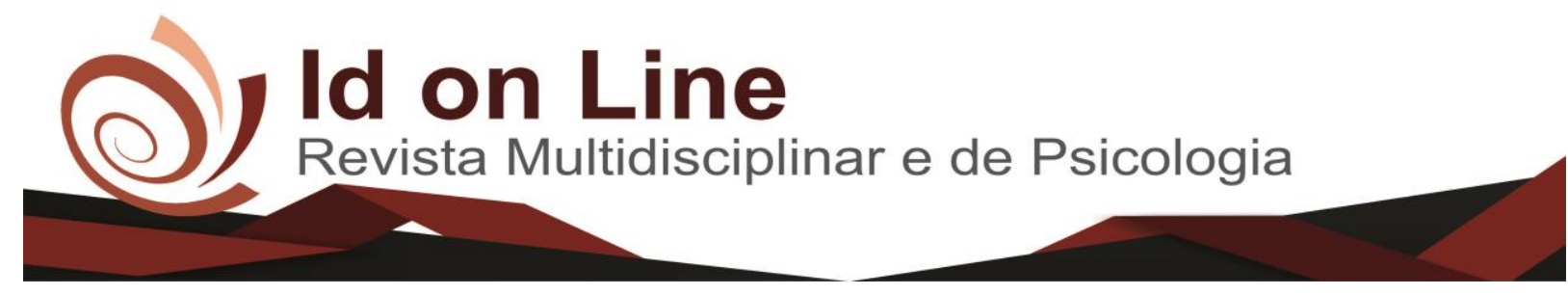

Artigo

\title{
Percepção dos Idosos \\ Acerca da Atividade Física na Terceira Idade
}

Naiara Santiago Ferraro ${ }^{1}$; Aldrina da Silva Confessor Cândido ${ }^{2}$

Resumo: O presente estudo teve como objetivo conhecer a percepção dos idosos acerca da atividade física na terceira idade.Trata-se de um artigo de Revisão Integrativa da Literatura de 15 artigos selecionados mediante os seguintes critérios de inclusão: abordar a temática proposta, estar entre os anos de 2011 e 2017, publicados na integra, em português e gratuitos. Os resultados apontaram que, quem mais pratica atividade física são idosos entre 60 e 70 anos, com patologias associadas; a principal atividade é a caminhada, numa rotina de realização de três vezes semanais, tendo a saúde como principal motivação para aderir à atividade física e as condições financeiras como o principal motivo para não aderir. Os benefícios mais citados se referem à socialização e qualidade de vida. Considerou-se queos idosos percebem que,quanto mais aderem a prática de atividade física,melhor é sua qualidade de vida, considerando os aspectos fisiológicos e psicossociais.

Palavras - chave: Saúde.Qualidade de vida. Terceira idade. Idosos. Atividade física.

\section{Perception of the Elderly About Physical Activity in Old Age}

\begin{abstract}
The present study had as objective to know the perception of the elderly about the physical activity in the third age. It is an integrative bibliographical review article, which 15 met the research objectives with the following inclusion criteria: studies that address the proposed theme, between 2011 and 2017, articles in full available online in Portuguese and free of charge. The results showed that those who practice physical activity are elderly between 60 and 70 years old, with associated pathologies; the main activity is walking, in a routine of accomplishment three times weekly, having health as the main motivation to adhere to physical activity and financial conditions as the main reason not to join. The most cited benefits refer to socialization and quality of life. It was considered that the elderly perceive that the more they adhere to the practice of physical activity, the better their quality of life, considering the physiological and psychosocial aspects.
\end{abstract}

Keywords: Health.Quality of life.Third age. Elderly. Physical activity.

\section{Introdução}

Sabe- se que o ser humano nasce, cresce, reproduz e morre entendendo que durante todos esses períodos da vida existem fases a serem passadas, uma delas é o envelhecimento, onde o mesmo se faz como um processo gradual e progressivo na vida de um indivíduo, entretanto podemos compreender que o envelhecimento se destaca por ser uma palavra comprida e pesada para ser carregada pelo ser humano, ao longo dos anos, destacando-se que

\footnotetext{
${ }^{1}$ Discente do curso de Enfermagem da Faculdade Independente do Nordeste. E-mail: nsferraro33@gmail.com;

${ }^{2}$ Docente da Faculdade Independente do Nordeste, doutoranda em Humanidades e Arte com ênfase em Ciências da Educação. E-mail: aldrina@fainor.com.br.77991974145.
} 
cada um tem sua forma particular e distinta de envelhecer. Além disso em todo o mundo cada dia mais com o passar do tempo cresce o número de pessoas com 60 anos, provavelmente esse número vem crescendo devido a redução da taxa de fertilidade e o crescimento da longevidade nos últimos tempos.

Afinal o envelhecimento é uma das fases da vida onde o corpo e a mente se modifica, com o passar dos anos, o indivíduo se apresenta fisicamente com as funções diminuídas, necessitando então a busca de atividades que contribuam para uma melhor expectativa de vida. É justamente nessa fase em que há uma perda cognitiva e uma diminuição das funções físicas, facilitando o surgimento de doenças que possam debilitar o idoso, gerando a perda das alterações fisiológicas. Em conformidade com tudo isso a sociedade vê o idoso como uma pessoa inútil e incapacitado de realizar várias funções simples do seu cotidiano, e principalmente, de serem inseridos no mercado de trabalho devido à perda das funções fisiológicas, claro associadas as doenças, como hipertensão, diabetes, problemas cardiovasculares, e perda da função neurológica, assim esses indivíduos são descartados de atividades que possivelmente teriam um melhor desempenho de pessoas mais novas.

Neste contexto, destaca-se a atividade física como possibilidade de oferecer uma melhor qualidade de vida na terceira idade. O sedentarismo e as incapacidades são fatores que podem interferir diretamente na qualidade de vida, mas já a prática constante de atividades física, contribui para uma expectativa de vida a longo prazo, proporcionando uma mudança do estilo de vida que contribui na redução de características que prejudiquem o envelhecer de um modo geral (JUNIOR et. al., 2015).

De um modo geral, para qualquer indivíduo, a realização de práticas de atividades físicas faz com que o corpo se sinta mais disposto e que a função e habilidades se tornem melhores. Em relação aos idosos, a realização da atividade física regular, resultará na melhora e diminuição de doenças que se classificam/apresentam de acordo o estilo de vida, contudo, proporcionando ao idosos uma vida mais ativa (JUNIOR, et. al., 2015).

Espera-se, com a atividade física uma melhoria da capacidade funcional e das habilidades físicas, o que gera uma diminuição das taxas de dependência para realização das atividades de vida diária (AVDs), promove uma expectativa de longevidade, pois melhora a percepção cognitiva, o estado mental e os níveis de humor, o que contribui para uma diminuição da depressão e consequentemente a inclusão em atividades, fazendo com que não ocorra o isolamento (JUNIOR, et. al., 2015). 
O trabalho se justifica pela necessidade de proporcionar aos idosos uma melhor qualidade de vida e um envelhecimento ativo, respeitando as suas limitações fisiológicas ou patológicas, ressaltando também que a atividade física poderá proporcionar aos idosos um melhor convívio social com outras pessoas.

Do ponto de vista de que o idoso tem que se movimentar de que não pode ficar parado, a prática de atividade física regularmente contribuirá para uma vida mais ativa e ajudará numa melhor qualidade de vida, melhorando cada dia mais as suas funções físicas, mentais e sociais. Podemos correlacionar e comparar que o jovem ativo de ontem, consequentemente, será um idoso ativo dos dias de hoje, fazendo com que a longevidade cresça mais e mais com o passar dos tempos, se tornando assim um ciclo positivo para as próximas gerações. Em virtude disso,este estudo teve como objetivo principal conhecer a percepção dos idosos acerca da atividade física, conhecendo a rotina da prática de atividade física de cada idoso,quais as atividades físicas são mais realizadas,descrevendo as motivações que levaram os idosos a aderirem ou não a atividade física e os impactos desta na vida dos idosos.

\section{Metodologia}

Quanto aos objetivos a pesquisa caracterizou-se como descritiva e exploratória de abordagem qualitativa. Segundo Gil (2010), as pesquisas descritivas têm como objetivo primordial a descrição das características de determinada população ou fenômeno ou, então, o estabelecimento de relações entre variáveis. Já sobre a pesquisa exploratória Marconi e Lakatos (2011) destacam que este tipo permite uma maior familiaridade entre o pesquisador e o tema pesquisado, visto que este ainda é pouco conhecido, pouco explorado.A pesquisa qualitativa é aquela que depende de fatores como a forma de coleta de dados, envolve categorização dos dados, interpretação destes e relatório escrito (GIL, 2010).

Quanto ao procedimento de coleta de dados, classificou-se como pesquisa bibliográfica. Foi escolhido o método da Revisão Integrativa" que é um método que proporciona a síntese de conhecimento e a incorporação da aplicabilidade de resultados de estudos significativos na prática" (SOUZA et.al., 2009). Para tanto, foram adotadas as seis etapas indicadas apara elaboração da revisão integrativa: 
1) Elaboração da pergunta norteadora: Para que a nossa pesquisa se organizassem e não abrisse viés para nenhum outro tema, utilizamos a seguinte pergunta: Qual a percepção dos idosos acerca da atividade física?

2) Busca ou amostragem da literatura:foram consultadas as bases de dados seguindo os seguintes critérios de inclusão: artigosque abordassem a temática proposta, com os descritores atividade física + idosos, entre os anos de 2011 e 2017, publicadosna integra, gratuitos e em português. No Google Acadêmico foram identificados 445 artigos e no Scielo (Scientific Electronic Library online) 65 artigos, dos quais, após filtrar pelos critérios acima citados, apenas 15 atenderam aos objetivos da pesquisa.

3) Coleta de dados: Para seleção e coleta de dados nos artigos selecionados, elaboramos um roteiro para nortear a presente pesquisa, buscando conhecer:

- Faixa etária dos idosos que praticam atividade física;

- Patologias mais comuns entre os idosos que praticam atividade física;

- Rotina de atividade física;

- As atividades mais realizadas por eles;

- Motivos pelos quais aderiram a prática de atividade física;

- Motivos para não adesão á prática da atividade física;

- Impactos da atividade física na vida dos idosos.

4) Análise critica dos estudos incluídos: Procedeu-se a leitura exaustiva dos artigos e o levantamento das informações, compondo um corpo textual que foi submetido ao Software Wordle (2014)para criar as nuvens de palavras ${ }^{3}$.

5) Discussão dos resultados: As informações obtidas foram analisadas e discutidas a luz do referencial teórico pertinente.

6) Apresentação da revisão integrativa: Descrição dos resultados, com as respectivas representações em quadros e figuras, bem como a discussão, utilizando o Microsoft Office Word, versão 15.26 e o Software Wordle (2014).

${ }^{3}$ www.wordle.net 


\section{Resultados e Discussão}

O quadro a seguir descreve as informações básicas dos quinze artigos analisados, dos quais, onze tiveram o estudo de campo como metodologia e quatro a revisão bibliográfica, publicados no período de 2011 até 2017. A temática central dos artigos era a atividade física, sendo abordado a faixa etária em que os idosos mais realizam tais atividades, a rotina de realização, as patologias destes idosos, as motivações para adesão e não adesão ao tratamento, bem como, os benefícios da realização de atividade física na terceira idade (QUADRO 1).

Quadro 1. Artigos selecionados nas bases de dados sobre atividade física na terceira idade.

\begin{tabular}{|c|c|c|c|c|}
\hline TITULO & OBJETIVOS & METODO & REVISTA & ANO \\
\hline $\begin{array}{l}\text { Exercícios Físicos e a Fatores } \\
\text { de Quedas em Idosos. }\end{array}$ & $\begin{array}{l}\text { Revisar e analisar quais } \\
\text { exercícios físicos são mais } \\
\text { efetivos para diminuir o } \\
\text { risco de quedas na } \\
\text { população idosa. }\end{array}$ & $\begin{array}{l}\text { Revisão } \\
\text { Bibliográfica }\end{array}$ & $\begin{array}{l}\text { VOOS Revista } \\
\text { Polidisciplinar } \\
\text { Eletrônica } \\
\text { Faculdade } \\
\text { Guairacá. }\end{array}$ & 2011 \\
\hline $\begin{array}{l}\text { Nível de Atividade Física } \\
\text { Habitual e Transtornos } \\
\text { Mentais Comuns entre } \\
\text { Idosos Residentes em Áreas } \\
\text { Rurais. }\end{array}$ & $\begin{array}{l}\text { Analisar a associação entre } \\
\text { o nível de atividade física } \\
\text { habitual e transtornos } \\
\text { mentais comuns entre } \\
\text { idosos. }\end{array}$ & $\begin{array}{l}\text { Estudo } \\
\text { Campo }\end{array}$ & $\begin{array}{l}\text { Revista Brasileira de } \\
\text { Geriatria e } \\
\text { Gerontologia. }\end{array}$ & 2014 \\
\hline $\begin{array}{l}\text { Influência da diabetes e a } \\
\text { prática de exercício físico e } \\
\text { atividades cognitivas e } \\
\text { recreativas sobre a função } \\
\text { cognitiva e emotividade em } \\
\text { grupos de terceira idade. }\end{array}$ & $\begin{array}{l}\text { Identificar atividades de } \\
\text { exercício físico que } \\
\text { envolvam atividades } \\
\text { cognitivas e recreativas, } \\
\text { sobre a função cognitiva e } \\
\text { aspectos emocionai de } \\
\text { idosos diabéticos e não } \\
\text { diabéticos. }\end{array}$ & $\begin{array}{l}\text { Estudo } \\
\text { Campo }\end{array}$ & $\begin{array}{l}\text { Revista Brasileira de } \\
\text { Geriatria e } \\
\text { Gerontologia. }\end{array}$ & 2014 \\
\hline $\begin{array}{l}\text { Atividade física na velhice: } \\
\text { avaliação de um grupo de } \\
\text { idosas sobre seus benefícios }\end{array}$ & $\begin{array}{l}\text { Identificar os benefícios da } \\
\text { prática da atividade física } \\
\text { no processo de } \\
\text { envelhecimento a partir da } \\
\text { experiência dos idosos } \\
\text { participantes do grupo Viva } \\
\text { Bem, do município de } \\
\text { Naque em Minas Gerais }\end{array}$ & $\begin{array}{l}\text { Estudo } \\
\text { Campo }\end{array}$ & $\begin{array}{l}\text { Revista } \\
\text { Enfermagem } \\
\text { Integrada }\end{array}$ & 2015 \\
\hline $\begin{array}{l}\text { Influência da atividade física } \\
\text { em grupo na qualidade de } \\
\text { vida e variáveis } \\
\text { cardiorrespiratórias em } \\
\text { idosos institucionalizados. }\end{array}$ & $\begin{array}{l}\text { Avaliar os efeitos da } \\
\text { atividade física sobre a } \\
\text { qualidade de vida de idosos } \\
\text { institucionalizados. }\end{array}$ & $\begin{array}{l}\text { Estudo } \\
\text { Campo }\end{array}$ & $\begin{array}{l}\text { FisiSenectus } \\
\text {.Unochapecó, Ano } \\
4 .\end{array}$ & 2016 \\
\hline $\begin{array}{l}\text { Motivação na prática de } \\
\text { atividade física para idosos } \\
\text { participantes do programa } \\
\text { vida saudável em } \\
\text { Parintins/AM. }\end{array}$ & $\begin{array}{l}\text { Identificar quais os motivos } \\
\text { que levam os idosos a } \\
\text { aderirem e permanecerem } \\
\text { em um programa regular de } \\
\text { exercício físico denominado }\end{array}$ & $\begin{array}{l}\text { Estudo } \\
\text { campo }\end{array}$ & $\begin{array}{l}\text { Coleção Pesquisa } \\
\text { em Educação Física } \\
\text { - Vol. 15, n. 2, } 2016\end{array}$ & 2016 \\
\hline
\end{tabular}




\begin{tabular}{|c|c|c|c|c|}
\hline & $\begin{array}{l}\text { vida saudável na cidade de } \\
\text { Parintins/AM. }\end{array}$ & & & \\
\hline $\begin{array}{l}\text { Prevalência da prática de } \\
\text { atividade física e correlação } \\
\text { coma saúde de idosos de } \\
\text { uma cidade no sul do brasil. }\end{array}$ & $\begin{array}{l}\text { Avaliar a prevalência de } \\
\text { idosos praticantes de } \\
\text { atividade física } \\
\text { em Caxias do Sul, RS, Brasil } \\
\text { e também a saúde desse } \\
\text { grupo. }\end{array}$ & $\begin{array}{l}\text { Estudo } \\
\text { Campo }\end{array}$ & $\begin{array}{l}\text { Revista Brasileira de } \\
\text { Reabilitação e } \\
\text { Atividade Física, }\end{array}$ & 2016 \\
\hline $\begin{array}{l}\text { Os benefícios da atividade } \\
\text { física para a qualidade de } \\
\text { vida dos idosos e a interação } \\
\text { social }\end{array}$ & $\begin{array}{l}\text { Demonstrar a importância } \\
\text { da atividade física na vida } \\
\text { do idoso, sua socialização. }\end{array}$ & $\begin{array}{l}\text { Revisão } \\
\text { Bibliográfica }\end{array}$ & $\begin{array}{l}\text { simpósio de TCC e } \\
\text { seminário de IC }\end{array}$ & 2016 \\
\hline $\begin{array}{l}\text { Fatores motivacionais } \\
\text { relacionados à prática de } \\
\text { atividades físicas em idosos }\end{array}$ & $\begin{array}{l}\text { Investigar os fatores } \\
\text { motivacionais de idosos } \\
\text { para praticarem atividades } \\
\text { físicas regularmente. }\end{array}$ & $\begin{array}{l}\text { Estudo } \\
\text { Campo }\end{array}$ & $\begin{array}{l}\text { Psicologia em } \\
\text { estudo, Maringá }\end{array}$ & 2016 \\
\hline $\begin{array}{l}\text { Atividade física de idosos e a } \\
\text { promoção da saúde nas } \\
\text { unidades básicas. }\end{array}$ & $\begin{array}{l}\text { Identificar e descrever as } \\
\text { ações de promoção a saúde } \\
\text { relacionadas a atividades } \\
\text { física de idosos nas } \\
\text { unidades básicas de saúde, } \\
\text { assim como a percepção } \\
\text { dos responsáveis sobre a } \\
\text { pratica. }\end{array}$ & Estudo de Caso & $\begin{array}{l}\text { Trab. Educ. Saúde. } \\
\text { Rio de Janeiro }\end{array}$ & 2016 \\
\hline $\begin{array}{l}\text { Bullyingna terceira idade: há } \\
\text { ocorrência naprática de } \\
\text { atividade física? }\end{array}$ & $\begin{array}{l}\text { Verificar se há ocorrência } \\
\text { de bullying na terceira } \\
\text { idade na prática da } \\
\text { atividade física. }\end{array}$ & $\begin{array}{l}\text { Estudo } \\
\text { Campo }\end{array}$ & $\begin{array}{l}\text { Coleção Pesquisa } \\
\text { em Educação Física }\end{array}$ & 2016 \\
\hline $\begin{array}{l}\text { Qualidade de vida de } \\
\text { pessoas idosas praticantes e } \\
\text { não praticantes de } \\
\text { atividades físicas. }\end{array}$ & $\begin{array}{l}\text { Avaliar a qualidade de vida } \\
\text { e a importância atribuída à } \\
\text { atividade física de pessoas } \\
\text { idosas. }\end{array}$ & $\begin{array}{l}\text { Estudo } \\
\text { Campo }\end{array}$ & $\begin{array}{ll}\text { Revista } & \text { do } \\
\text { departamento } & \text { de } \\
\text { Psicologia } & \end{array}$ & 2017 \\
\hline $\begin{array}{l}\text { Relação entre atividade } \\
\text { física e depressão em } \\
\text { idosos: uma revisão de } \\
\text { literatura. }\end{array}$ & $\begin{array}{l}\text { Buscou avaliar a relação } \\
\text { entre a prática de } \\
\text { atividades físicas e } \\
\text { aredução se sintomas } \\
\text { depressivos }\end{array}$ & $\begin{array}{l}\text { Revisão } \\
\text { Bibliográfica }\end{array}$ & $\begin{array}{l}\text { Corpo, consciência, } \\
\text { Cuiabá MT, }\end{array}$ & 2017 \\
\hline $\begin{array}{l}\text { Abandono do idoso à } \\
\text { atividade física: Uma revisão } \\
\text { de literatura }\end{array}$ & $\begin{array}{l}\text { Evidenciar e discutir quais } \\
\text { os principais motivos que } \\
\text { levam os idosos a não } \\
\text { aderirem ou abandonar } \\
\text { programas específicos de } \\
\text { atividade física voltados } \\
\text { para essa faixa etária. }\end{array}$ & $\begin{array}{l}\text { Revista Saúde e } \\
\text { Desenvolviment } \\
\text { o }\end{array}$ & $\begin{array}{l}\text { Revisão } \\
\text { Bibliográfica }\end{array}$ & 2017 \\
\hline
\end{tabular}




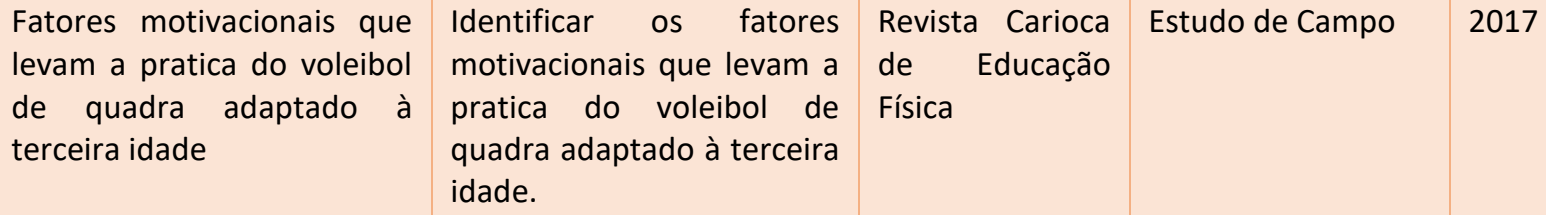

Fonte: Dados da pesquisa (2017).

\section{Faixa Etária Predominante}

Dentre os artigos selecionados foram encontrados relatos de diversas faixas etárias de idosos praticantes de atividade física, mas entre elas uma foi predominante, a de idosos entre os 60 e 70 anos de idade, sendo menos citados os idosos mais longevos.

Sabe-se que, com o avançar da idade os idosos se tornam mais dependentes e, consequentemente, acontece um processo de perdas, desenvolvendo um sentimento de incapacidade que parece ser um fator desencadeador para o desinteresse pelas atividades cotidianas e também pela prática de atividade física (DALVINO 2016).

\section{Patologias Associadas}

A análise dos artigos apontou que as patologias mais recorrentes e corriqueiras na vida dosidosos, mesmo dos praticantes de atividade física, são: a hipertensão, o diabetes, a osteoporose e a depressão. Salienta-se que tais patologias não são obstáculos para a realização de atividade física, já que podem ser reversíveis e controláveis com mudança de hábitos alimentares e com a prática da atividade física. Conforme Ferreira (2015), a atividade física pode ser realizada com programas de exercícios de forma individual, considerando a necessidade de cada idoso e a patologia associada; contribuindo para a redução e prevenção das dores corporais, da hipertensão, do diabetes, da depressão, além de proporcionar um bem-estar físico e mental para a saúde dos idosos. 


\section{Rotina de Atividade Física}

Os artigos apontam que a prática de atividades física acontece de uma a cinco vezes por semana, sendo que a rotina de realização de exercício três vezes por semana prevaleceu. Este dado esta de acordo com as recomendações da Organização Mundial de Saúde - OMS que preconiza, para pessoas acima de 65 anos de idade, uma média de 150 minutos de atividade física de intensidade moderada ou 75 minutos de atividade vigorosa. Considera-se quea atividade moderada, como caminhar, pedalar e praticar esportes, tem benefícios significativos para saúde dos idosos. Já em casos de idosos que apresentam pouca mobilidade a recomendação é de atividades físicas que melhorem o equilíbrio e previnam as quedas sendo realizadas três ou mais dias por semana (OMS, 2017).

É muito importante manter os idosos cientes das recomendações quanto a rotina de atividade física, bem como, quanto aos benefícios da mesma, pois, como afirma Carvalho e Castellano (2016) esta ciência é um fator primordial e motivacional para a constante eficácia e permanência regular dos idosos na prática de atividade física.

\section{Atividades Físicas Mais Realizadas}

A caminhada se destacoucomo atividade física mais praticada entre os idosos conforme as informações dos artigos, seguido do alongamento, da hidroginástica, exercícios de musculação, atividades de dança e de bicicleta (FIGURA 1). 


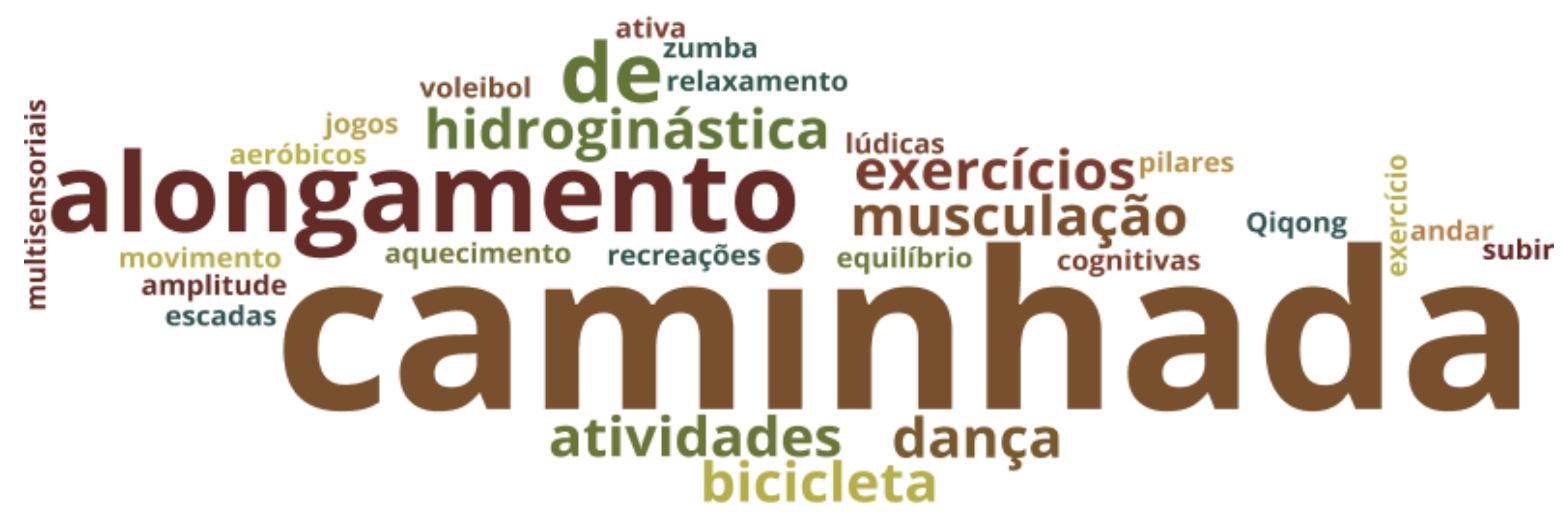

Figura 1: Representação das atividades físicas mais realizadas pelos idosos (Dados da pesquisa, 2017).

A importância da caminhada para os idosos foi reafirmada em um estudo desenvolvido na Estratégia Saúde da Família - ESF de Grajaú - MA, onde buscou-se conhecer na óptica dos idosos os efeitos da prática da caminhada. O estudo concluiu que as caminhadas, conforme relato dos idosos, contribuíam para o controle das doenças crônico degenerativas para o alivio dos quadros álgicos, redução da massa corpórea, melhora na saúde mental e social e melhora na mobilidade física (TORRES et al., 2013).

\section{Motivações para Adesão à Atividade Física}

$\mathrm{Na}$ maioria dos artigos analisados, os idosos relatam que, o que os levou a fazer a atividade física foi a questão saúde, seguido de prazer, autoestima, indicação médica, socialização,convite, a prevenção e a estética, dentre outros motivos menos citados(FIGURA2). 


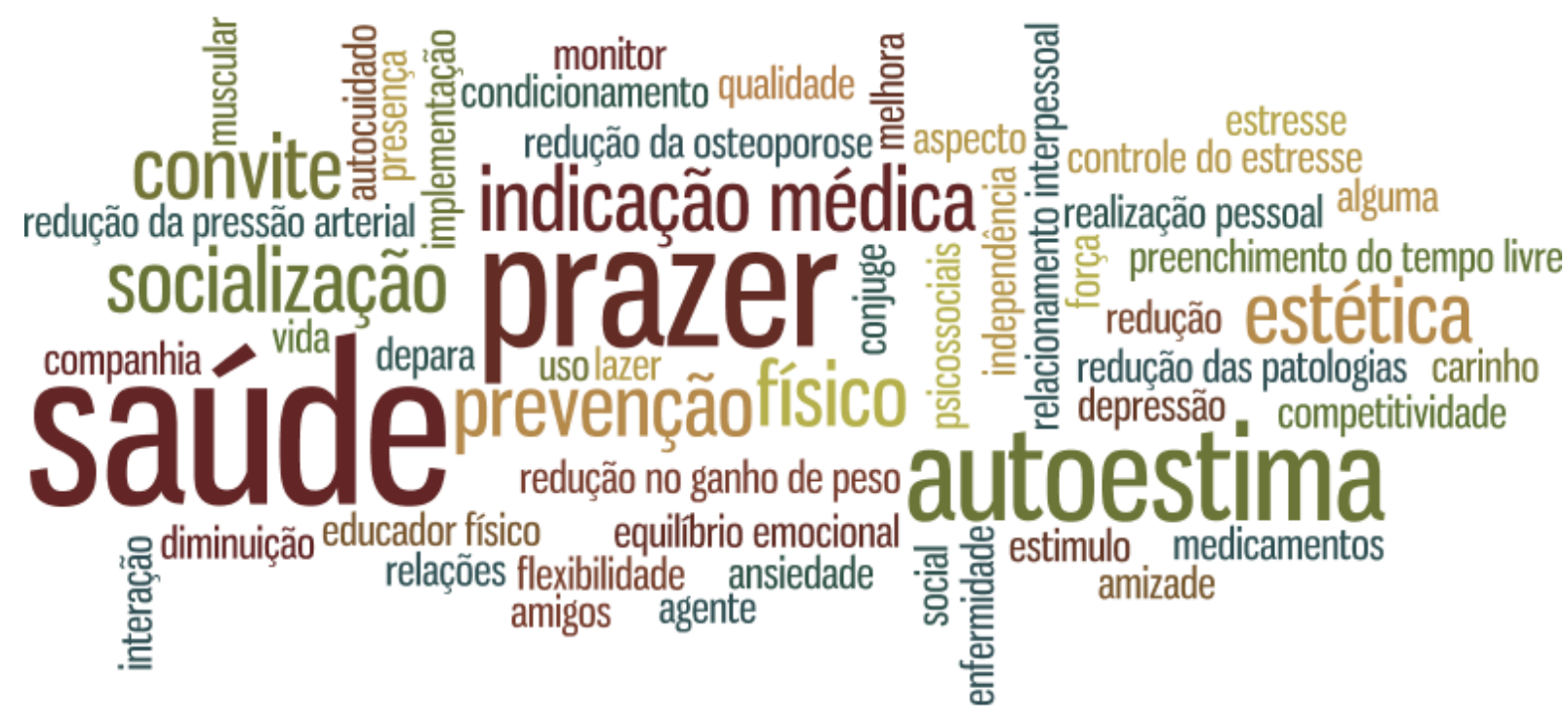

Figura 2: Representação dos motivos que contribuem para adesão à atividade física pelos idosos (Dados da pesquisa 2017).

Segundo Bueno et al., (2013) há uma inversão dos motivos para a adesão á atividade fisica entre os adultos mais jovens - questões estéticas - e os idosos - saúde. Os dados da presente pesquisa corroboram com esta informação já que o fator estética foi menoscitado quando comparado ao fator saúde. Com o passar dos anos, as pessoas idosas vão diminuindo essa vontade de cuidar só do externo, passando a se preocupar exclusivamente com a sua saúde e seu bem-estar (SEPULCHRO, 2017).

Vê-se que, nesta fase da vida, há uma menor preocupação com o aspecto estético e uma maior importância à saúde. Como afirma Sepulchro (2017), nos dias atuais a prática de atividade física na terceira idade está sendo muito constante, e a maior preocupação dessa categoria está sendo mesmo a manutenção da saúde e a independência funcional, seguido de uma melhoria na qualidade vida e a socialização com outras pessoas. A atividade física, além de melhorar os problemas de saúde como, hipertensão, diabetes, osteoporose, vem a contribuir principalmente para a interação social dos idosos com pessoas da sua mesma faixa etária e também com os mesmos pensamentos compatíveis à idade. Segundo Ferreira (2015) foi 
percebido que a atividade física, realizada em grupo, contribuiu ainda mais para a adesão e aceitação dos idosos beneficiando a sua saúde em todos os aspectos possíveis.

\section{Motivações para Não Adesão à Atividade Física}

É notório que, os idosos enfrentam dificuldades e limitações para a realização de atividade física. Dentre os fatores relatados como que os impediam de realizar alguma atividade física se destacou as condições financeiras, seguido de falta de tempo, cansaço,a falta de companhia, dentre outros (FIGURA 3).

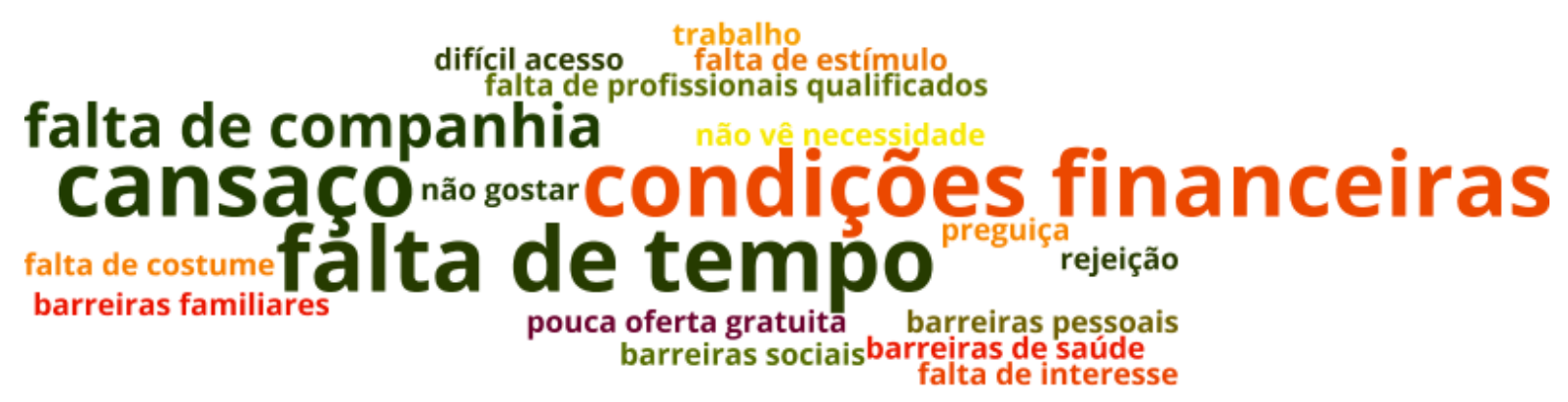

Figura 3: Representação dos motivos que contribuem para a não adesão dos idosos à atividade física (Dados da pesquisa, 2017).

Apesar dos idosos terem citado a caminhada como a principal atividade fisica realizada, conforme discutido anteriormente, suponhe-se que há um risco para a inatividade fisica por conta dos motivos para não adesão acima citados. De acordo com a OMS (2014), a inatividade fisica é o quarto principal fator de risco de mortalidade global, além de ser causa predisponente para ocorrência dos canceres, da diabetes e das doenças cardiacas isquemicas. Portanto, é 
primordial que os profissionais de saúde, em particular os enfermeiros, incentivem os idosos á prática da atividade fisica, buscando conhecer e intervir nas suas principais limitações.

\section{Impactos da Atividade Física}

Uma grande parte dos artigos analisados versam sobre a temática dos impactos causados pela atividade física nos idosos, sendo encontrado nesta análise, apenas os impactos positivos, tais como, a socialização, melhora na qualidade de vida, melhora no peso, melhora no humor, impacto psicológico, impacto no convívio com outras pessoas, redução de quedas e fraturas (FIGURA 4).

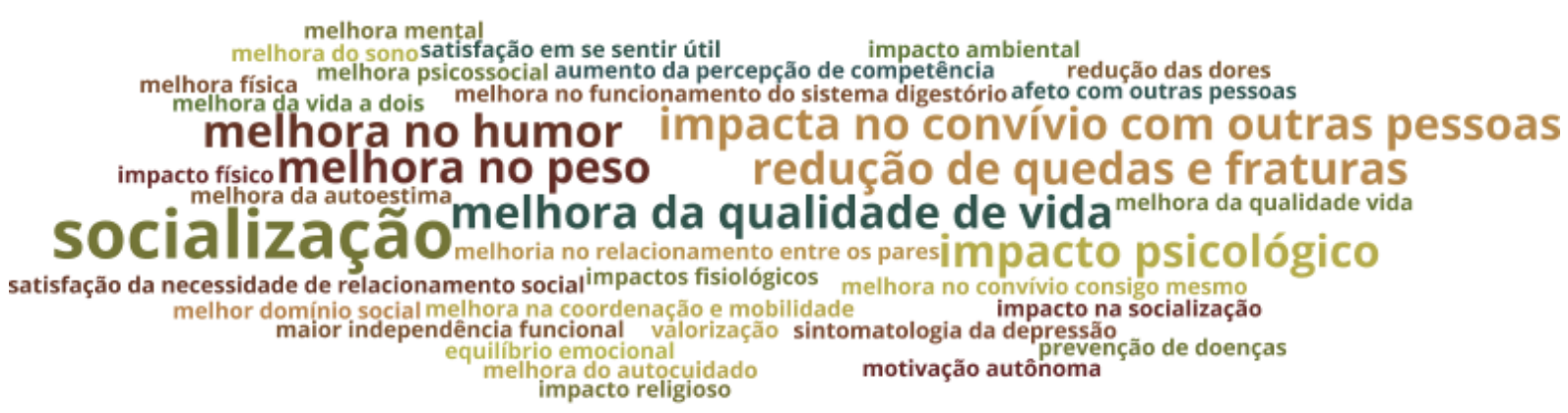

Figura 4: Representação dos impactos da atividade física na vida dos idosos (Dados da pesquisa, 2017).

De acordo com a OMS (2017), idosos fisicamente ativos possuem menor risco para doenças coronarianas, acidente vascular encefálico, hipertensão, diabetes, câncer de colón e de mama; apresentam melhor condicionamentocardiorrespiratório e muscular e composição e massa corpórea mais saudável. Além disso,previne o organismo de doenças cardiovasculares, 
diabetes e proporciona maior equilíbrio corporal, melhorando a capacidade funcional, diminuindo o risco de quedas e melhorando a função cognitiva.

Segundo Junior (2012) a prática de atividade física tem um impacto enorme na vida desses idosos, pois traz consigo vários benefícios psicossociais, além dos biológicos, entre eles o enfrentamento dos problemas e dos desafios vividos diariamente por eles, ressaltando que a atividade física contribui veemente para que os idosos não tenham um sentimento de perda constante no decorrer de suas vidas.

\section{Considerações Finais}

A Revisão Integrativa nos permitiu conhecer a percepção dos idosos quanto a atividade física. Dentre os dados mais importantes destacaram-se a idade dos idosos que mais realizam atividade física, prevalecendo dos 60 aos 70 anos, mesmo possuindo patologias associadas como hipertensão, diabetes, osteoporose e depressão. A rotina de atividade física é no geram de três vezes semanais sendo a atividade mais realizada a caminhada.

A análise dos artigos permitiu-nos apreender que, na visão dos idosos, a saúde é o principal fator motivador para adesão à atividade física e, as condições financeiras o principal motivo da não adesão, associados a outros fatores, o que pode trazer um risco de inatividade física para a população idosa. Já dentre os impactos da atividade física na terceira idade, os principais relatados foram a socialização e a melhoria da qualidade de vida.

Ao final, considera-se que, a prática de atividade física na terceira idade é muito importante para a manutenção da saúde e prevenção de algumas doenças crônicas na vida dos idosos, contribuindo para a superação das limitações advindas com o envelhecimento, melhorando a capacidade funcional, independência pessoal e promovendo a socialização.Idosos motivados pela prática de atividade física são muito mais motivados para melhora da saúde, melhora do humor, da qualidade de vida e da socialização com outras pessoas.

Cabe ao profissional de saúde estimular a prática da atividade física pelos idosos, reconhecendo as limitações e possibilidades de cada um, proporcionando um envelhecimento ativo, fazendo com que os idosos se sintam úteis e felizes devido a prática da atividade física. 


\section{Referências}

BUENO, Denise et al. Caminhada e Orientação Profissional: associações com conhecimento das recomendações internacionais e Motivo auto - referido. Educação Física em Revista, v. 7, n. 3, p. $74-85,2013$

CARVALHO, Jessica Belem et al. Motivação na Prática de Atividade Física para IdososParticipantes do Programa Vida Saudável em Parintins/AM. Coleção Pesquisa em educação Física, v.15, n. 2, p.135 - 142, 2016.

CONSTANTINI, Amanda et al. Exercícios Físicos e Fatores de Quedas em Idosos. VOOS Revista Polidisciplinar Eletrônica da Faculdade Guariacá, v.3, n. 2, p.17-30, 2011.

DALVINO, Jéssica Cristrina et al. Os Benefícios da Atividade Física para a Qualidade de Vida dos Idosos e a Interação social. Simpósio de TCC e Seminário. p. 900-908 IC,2016/1.

FELICIANO, Angelica Fernanda. et al. Bullying na Terceira Idade: Ocorrência na Prática de Atividade Física? Coleção Pesquisa em Educação Física, Várzea Grande - Sp, v. 15, n. 4, p.41-50, 2016.

FERREIRA, Taciany Karine de Almeida et al. Atividade Física na Velhice: Avaliaçãode um Grupo de Idosassobre seus Benefícios. Revista Enfermagem Integrada, Ipatinga - Mg, v. 1, p.1303-1313, 2015.

GIL, Antônio Carlos. Como elaborar projetos de pesquisa. Edição 5. Editora Atlas, 2010.

JUNIOR, José Roberto Andrade do NAscimento et al. Impacto na Prática de Atividade Física no Estresse Percebido e na Satisfação de Vida de Idosos. Rev.Educ. Fis/UEM, v. 23, n. 4, p. 647-654, 2012.

JÚNIOR, Vicente F. F. G.; BRANDÃO, Athus B.; ALMEIDA, Francisco J. M. de;

OLIVEIRA, Janaísa G. D. de. Compreensão de Idosos sobre os Benefícios da Atividade Física, Volume 19, Número 3, Páginas 193-198, 2015. ISSN 1415-2177.

LAKATOS, E; MARCONI,M. Metodologia Cientifica.São Paulo: Atlas, 2007.

ORGANIZAÇÃO MUNDIAL DE SAÚDE. Atividade física - Folha Informativa n.385. 2014.

ORGANIZAÇÃO MUNDIAL DE SAÚDE. Physical Activity and Older Adults. 2017. Disponível em: http://www.who.int/dietphysicalactivity/factsheet olderadults/en/

PEREIRA, Diogo Fagundes. Relação entre Atividade Física e Depressão em Idosos: Uma Revisão de Literatura. Corpoconsciência, Cuiabá - MT, v.20, n.03, 22-28, 2016.

PETRY, Ana Laura Nicoletti et al. Influência da Atividade Física em grupos na qualidade de vida e variáveis cardiorespiratórias em idosos institucionalizados. Fisisinectus, São Paulo, v. 1, n. 4, p.23-31, 2016. 
PINTO, Lelia Lessa et al. Nível de Atividade Física Habitual e Transtornos Mentais comuns entre Residentes em Áreas Rurais. Revista Brasileira de Geriatria e Gerontologia, Rio de Janeiro, v. 17, n. 4, p. 819-828, 2014.

REIS, Camila Cristina et al. Prevalência da Prática de Atividade Física e Correlação com a saúde de idosos de uma Cidade no Sul do Brasil.Revista Brasileira de Reabilitação e Atividade Física, Vitória, v. 5, n. 01, p. 27-32, 2016.

ROSSI, Paulo Giusti. et al. Abandono do Idoso á Atividade Física: uma revisão de literatura. Revista Saúde e Desenvolvimento, São Paulo, v. 11, n. 6, p.259-263, 2017.

SÁ, Paulo Henrique Vilela Oliveira de. et al. Atividade Física de Idosos a Promoção de Saúde nas Unidades Básicas.Trabalho Educacional, Rio de Janeiro, v. 2, n. 14, p.545-558, 2016.

SEPULCHRO, Breno do Nascimento et al. Fatores Motivacionais que levam a Prática do Voleibol de Quadra Adaptado á Terceira Idade. Revista Carioca de Educação Física - Rio de Janeiro, v. 12, n. 01, p. 43-51, 2017.

SILVA, Agnes Navarro et al. Fatores Motivacionais Relacionados á Prática de Atividade Física em Idosos. Psicologia em Estudo, Maringá, v. 21, n. 04, p. 677-685, 2016.

SOUZA, William C. de; MASCARENHAS, Luis P. G.; GRZELCZAK, Marcos T.; JUNIOR, Douglas T.; BRASILINO, Fabrício F.; LIMA, Valderi A. de. Exercício físico na promoção da saúde na terceira idade, Saúde Meio Ambient. v. 4, n. 1, p. 55-65, jan./jun. 2015 ISSN 2316-347X.

TORRES, Ayslan Goncalves et al. Efeitos da Prática de Caminhada de Idosos em Grupo: um olhar do protagonista. J Manag Prim Health Care, v. 4, n. 1, p. 19-26, 2013.

VARGAS, Liane da Silva et al. Influência da Diabetes e a Prática de Exercício Físico e Atividades Cognitivas e Recreativas sobre a Função Cognitiva e Emotividade em Grupos de Terceira Idade.

Revista Brasileira de Geriatria e Gerontologia, Rio de Janeiro, v. 17, n. 4,p. 867-878, 2014.

WINTER, Julia et al. Qualidade de Vida de Pessoas Idosas Praticantes ou não Praticantes de Atividades Físicas. PSI UNISC, Santa Catarina do Sul, v.1,n.1, 89- 96, 2017.

\section{Como citar este artigo (Formato ABNT):}

FERRARO, Naiara S.; CÂNDIDO, Aldrina da S.C. Percepção dos Idosos Acerca da Atividade Física na Terceira Idade. Id on Line Revista Multidisciplinar e de Psicologia, 2017, vol.11, n.38, p.597611. ISSN: 1981-1179.

Recebido: 01.10.2017

Aceito: 03.11.2017 